thought to be an integral part of chemoresistance, but the relation of these adaptations to chemoresistance is poorly understood. Our aim was to identify the metabolic adaptations that are specifically associated with platinum-resistant (PR) cell lines and its platinum-sensitive (PS) derivatives across multiple OC cell lines.

Methods Targeted metabolic analysis evaluating 242 metabolites of the PS A2780, PEO1, and mR182 cell lines was performed along with their respective PR derivatives, C200, PEO4, R182. The group comparison was performed using unpaired t-tests followed by FDR correction. The differentially expressed metabolites were identified using two criteria: FDR $\leq 5 \%$ and absolute fold-change $\geq 1.5$. The pathway analysis was performed using Metaboanalyst ${ }^{\mathrm{TM}}$ with the metabolites that have unadjusted $\mathrm{p}$-value $\leq 5 \%$.

Results Many significantly impacted pathways were conserved among the PR cell lines. Compared to the PS counterparts, the PR PEO4, C200, and R182 lines had metabolite concentrations with $\mathrm{FC} \geq 1.5$ in 29,44 , and 28 measured metabolites, respectively. The top pathways impacted were 'nicotinate and nicotinamide metabolism', 'purine metabolism', and 'phenylalanine, tyrosine, tryptophan biosynthesis'. A global analysis of PS vs PR was performed. The top five significantly impacted pathways were: Arginine biosynthesis, Pyrimidine and Purine metabolism, Phenylalanine, tyrosine and tryptophan biosynthesis' and 'Starch and sucrose metabolism'.
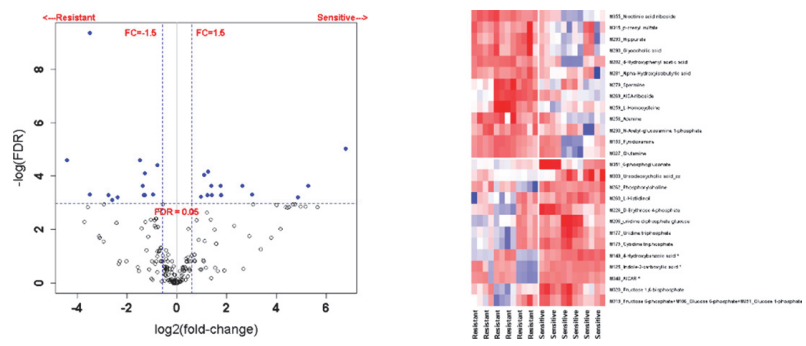

Abstract EPV002/\#308 Figure 3 a) Volcano plot of sensitive cohort vs resistant cohort; b) Heat map of sensitive cohort vs resistant cohort

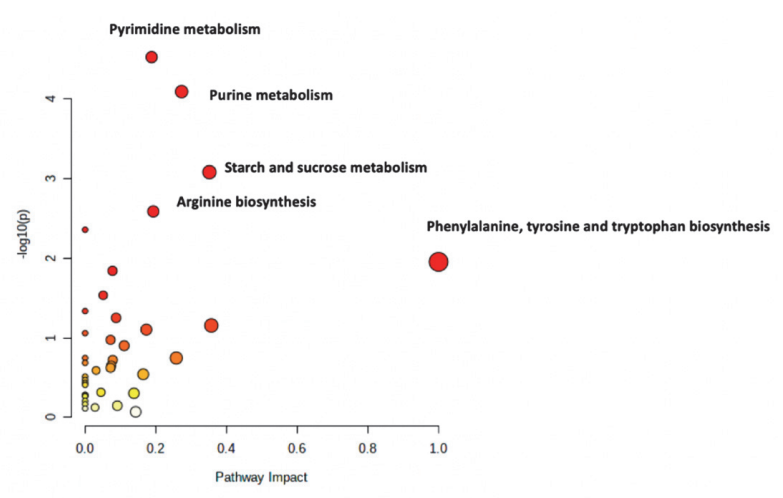

Abstract EPV002/\#308 Figure 4 Top impacted pathways of combined PR cohort compared to PS cohort

Conclusions We identified multiple shared metabolomic pathways among established PR OC cell lines that highlight conserved motifs of PR. These may represent targetable pathways to predict or reverse chemoresistance.

\section{EPV003/\#326 AN INTEGRATED GENOMIC, PROTEOMIC AND IMMUNOPEPTIDOMIC APPROACH TO DISCOVER NOVEL TUMOUR NEOANTIGENS IN AN IMMUNOLOGICALLY COLD OVARIAN CANCER FOR PERSONALISED T-CELL RECEPTOR THERAPY}

${ }^{1,2} \mathrm{GY} \mathrm{Ho}{ }^{*},{ }^{3} \mathrm{P}$ Faridi, ${ }^{1} \mathrm{~J} \mathrm{Wu},{ }^{4,5} \mathrm{H}$ Barker, ${ }^{1,6} \mathrm{~T}$ Nguyen-Dumont, ${ }^{1} \mathrm{~J}$ Chang, ${ }^{1} \mathrm{~A}$ Fell, ${ }^{1} \mathrm{P}$ Eggenhuizen, ${ }^{1} \mathrm{~J}$ Steen, ${ }^{2} \mathrm{~T}$ Manolitsas, ${ }^{1,2} \mathrm{~S}$ Frentzas, ${ }^{4,7} \mathrm{~J}$ Bedo, ${ }^{4,5} \mathrm{C}$ Vandenberg, ${ }^{4,5} \mathrm{~T}$ Papenfuss, ${ }^{4,5} \mathrm{C}$ Scott, ${ }^{1} \mathrm{~J}$ Ooi, ${ }^{1,2}$ E Segelov. 'Monash University, School of Clinical Sciences, Clayton, Australia; ${ }^{2}$ Monash Health, Oncology Department, Clayton, Australia; ${ }^{3}$ Monash University, Monash Biomedicine Discovery Institute, Clayton, Australia; ${ }^{4}$ Walter and Eliza Hall Institute of Medical Research, Cancer Biology and Stem Cells Division, Parkville, Australia; ${ }^{5}$ University of Melbourne, Department of Medical Biology, Parkville, Australia; ${ }^{6}$ University of Melbourne, 6. Departcomputing and Information Systems, Parkville, Australia; 'University of Melbourne, Department of Computing and Information Systems, Parkville, Australia

\subsection{6/ijgc-2021-IGCS.70}

Objectives Ovarian carcinosarcoma (OCS) are rare aggressive cancers with poor prognosis and limited effective treatments. The tumour mutation burden in OCS is often low. Therefore, these tumours are immunologically 'cold' and relatively irresponsive to single agent immunotherapy. We explored tumour neoantigen discovery in an OCS using various genomic and proteomic platforms for personalised T-cell receptor (TCR) therapy.

Methods Whole genome sequencing (WGS) was performed on SFRC01177 OCS tumour specimen taken at surgery. Fresh tumour specimens obtained at surgery and biopsy at recurrence were engrafted subcutaneously in NOD-scidIL2Rgammanull (NSG) to generate a paired patient derived xenograft (PDX) model. Whole exome sequencing and RNA sequencing (WES/RNAseq) together with nano-ultra-performance liquid chromatography coupled to high-resolution mass spectrometry were performed on the snap frozen tumours from the baseline and recurrent PDX for tumour neoantigen (TNA) discovery.

Results A total of 6,500 mutant TNA were predicted in silico from the baseline WGS data which were narrowed down to 65 and 33 respectively based on the baseline and recurrent PDX tumours WES/RNAseq data. The immunopeptidomic analysis revealed over 100 major histocompatibility complex bound antigens including mutant, spliced and cancer testis antigens. The PDX was re-established in NSG MHC ${ }^{\text {null }}$ mouse model and was shown to retain the platinum refractory in vivo response as well as to tolerate 1 million HLA-matched donor CD8 + T-cell injections.

Conclusions We have discovered multiple tumour specific neoantigens using the comprehensive TNA discovery platforms, which will direct our TCR engineering. In parallel, we have also established an OCS PDX model suitable for cell-based therapy testing.

\section{EPV004/\#360 INHIBITION OF CANCER CELL-DEPENDENT GLYCOLYSIS THROUGH AVB-500, A SELECTIVE INHIBITOR OF GAS6-AXL, IN COMBINATION WITH PACLITAXEL IN HIGH-GRADE ENDOMETRIAL CANCER}

${ }^{1} S$ Bruce*, ${ }^{1} \mathrm{E}$ Lomonosova, ${ }^{1} \mathrm{H}$ Noia, ${ }^{1} \mathrm{E}$ Stock, ${ }^{2} \mathrm{~K}$ Cho, ${ }^{1} \mathrm{D}$ Khabele, ${ }^{1} \mathrm{~L}$ Kuroki, ${ }^{1} \mathrm{~A}$ Hagemann, ${ }^{1} \mathrm{C}$ Mccourt, ${ }^{1} \mathrm{P}$ Thaker, ${ }^{1} \mathrm{D}$ Mutch, ${ }^{1} \mathrm{M}$ Powell, ${ }^{2} \mathrm{~L}$ Shriver, ${ }^{2} \mathrm{G}$ Patti, ${ }^{1} \mathrm{~K}$ Fuh. 'Washington University, Gynecologic Oncology, St. Louis, USA; ${ }^{2}$ Washington University, Chemistry, St. Louis, USA

10.1136/ijgc-2021-IGCS.71 
Objectives AXL is a receptor tyrosine kinase that is activated by GAS6. Overexpression of AXL is correlated with the glycolytic phenotype in metastatic lung cancer. Cancer cells preferentially convert glucose to lactate via glycolysis which promotes growth and survival. It is unknown whether inhibition of AXL can prevent glycolysis in endometrial cancer causing cell death. The aim of this study was to determine whether AVB-500 can increase sensitivity to paclitaxel through inhibition of glycolysis.

Methods Cell viability was performed with high-grade endometrial, chemo-resistant cell lines, ARK1 and PUC1. Cells were treated with paclitaxel (P) and with AVB-500+paclitaxel (AVB-500+P). Intraperitoneal ARK1 or PUC1 tumors were treated with vehicle, AVB-500, P, or AVB-500+P. Cell lysates were analyzed using the Jess system. A Seahorse Analyzer was used for glycolytic rate assays. Stable isotope tracing was used for in vivo metabolite abundance quantification.

Results We found that ARK1 and PUC1 cells had decreased viability when treated with $\mathrm{AVB}-500+\mathrm{P}$ than when treated with P alone. ARK1 and PUC1 in vivo IP models had significantly fewer tumors and decreased tumor weight when treated with AVB-500+P compared to P alone. Treatment with AVB$500+\mathrm{P}$ was found to decrease basal glycolysis in vitro through decreased AKT activation. Multiple glycolytic metabolites were decreased in the tumors of AVB-500 $+\mathrm{P}$ compared to treatment with $\mathrm{P}$ alone.

Conclusions We demonstrate that the addition AVB-500 to paclitaxel improves endometrial cancer chemo-sensitivity. We show that this therapeutic combination decreases basal glycolysis through reduced PI3K/AKT signaling. This provides a metabolic mechanism for increasing uterine cancer sensitivity to chemotherapy.

\section{EPV005/\#536 MOLECULAR ANALYSIS OF PRIMARY ENDOMETRIAL CANCER AND MATCHED LUNG METASTASES}

${ }^{1} S$ Gordhandas*, ${ }^{1} \mathrm{~A}$ Da Cruz Paula, ${ }^{2} \mathrm{E}$ Kertowidjojo, ${ }^{1} \mathrm{~K}$ Dessources, ${ }^{2} \mathrm{~A}$ Maroldi, ${ }^{2} \mathrm{E}$ Da Silva, ${ }^{2} \mathrm{~F}$ Derakhshan, ${ }^{1} \mathrm{~N}$ Abu-Rustum, ${ }^{1} \mathrm{~B}$ Weigelt. 'Memorial Sloan Kettering Cancer Center, Gynecology Service, Department of Surgery, New York, USA; ${ }^{2}$ Memorial Sloan Kettering Cancer Center, Pathology, New York, USA

\subsection{6/ijgc-2021-IGCS.72}

Objectives The molecular processes underpinning distant metastasis in endometrial cancer (EC) are not well understood. We sought to characterize the genomic alterations of primary ECs and matched lung metastases.

Methods Primary ECs, matched lung metastases, and normal tissue from two patients were subjected to whole-exome sequencing. Sequencing data were analyzed using validated bioinformatics tools.

Results In case 1 , sequencing analysis of the primary FIGO grade 2 EC and matched lung metastasis, which developed after 3 years, revealed 99 and 95 non-synonymous somatic mutations, respectively, of which 68 were shared. In addition to clonal shared PIK3R1 and PTEN mutations, we observed clonal shifts in the progression to lung metastatic disease, including a CTNNB1 G34V hotspot mutation, which was subclonal in the primary EC and became clonal in the metastasis. The primary dedifferentiated EC of case 2 was MSI-H due to MLH1 hypermethylation. Both the primary and synchronous metastatic lung tumor harbored a large number of somatic mutations (1217 and 1157, respectively), but only 227 were shared, including PIK3CA hotspot, PTEN frameshift, and MSH6 frameshift mutations. In case 2, clonal shifts were also observed in the progression from primary EC to lung metastasis, and multiple CTNNB1mutations were identified: a subclonal CTNNB1 G34R hotspot mutation in the primary EC, and a subclonal CTNNB1G33P hotspot and a clonal CTNNB1 G34E hotspot mutation limited to the metastasis.

Conclusions Clonal shifts, the accumulation of additional mutations and/or hotspot CTNNB1 mutations may play a role in the progression from primary EC to lung metastatic disease.

\section{EPV006/\#578 ROLE OF CHRONIC STRESS ON ANTI-TUMOR T- CELL RESPONSES IN OVARIAN CANCER}

${ }^{1} \mathrm{~A}$ Aquino-Acevedo*, ${ }^{2} \mathrm{H}$ Knochenhauer, ${ }^{1} \mathrm{M}$ Ortiz-León, ${ }^{1} \mathrm{Y}$ Rivera-López, ${ }^{1} \mathrm{M}$ BonillaClaudio, ${ }^{2} \mathrm{R}$ Previs, ${ }^{1} \mathrm{G}$ Armaiz-Pena. ${ }^{1}$ Ponce Health Sciences University, Department of Basic Sciences (pharmacology), Ponce, Puerto Rico; ${ }^{2}$ Duke University School of Medicine, Obstetrics and Gynecology, Durham, USA

\subsection{6/ijgc-2021-IGCS.73}

Objectives A cancer diagnosis increases stress hormones and leads to altered psychological states. Work from our team suggests that chronic stress promotes an increased inflammatory response. Preliminary data show an altered $\mathrm{CD} 4+/ \mathrm{CD} 8+\mathrm{T}-$ cell ratio and a heterogeneous expression of exhaustion markers in patients with high-grade serous ovarian cancer (HGSOC). Therefore, we hypothesized that chronic stress results in loss of effector T-cell response and increased exhaustion.

Methods We obtained ascites samples from 66 patients with HGSOC and measured cytokine levels using a comprehensive cytokine/chemokine magnetic bead panel. Metanephrine (an epinephrine metabolite) levels from ascites were measured by ELISA. CD8 + T-cells isolated from OC patient ascites were stimulated with epinephrine and flow cytometry was used to measure co-expression of CD38 activation marker and Granzyme B, an essential mediator of CD8 + T-cell killing capacity. Results Showed a significant increase in inflammatory cytokines in chemo-resistant and recurrent tumors: Eotaxin $(p \leq 0.002)$, IL-6 ( $\mathrm{p} \leq 0.003)$, and IL-7 ( $\mathrm{p} \leq 0.009)$. Metanephrine, was positively correlated with pro-tumoral and inflammatory cytokines: SCD40L $(p=0.032)$, FGF-2 $(p=0.033)$ and MIP1a $(\mathrm{p}=0.03)$. Ascites-derived CD8 + T-cells treated with epinephrine, showed a decreased co-expression CD38 and Granzyme B $(p=0.004)$. These results suggest a role for stress hormones in T-cell activity suppression.

Conclusions Chemo-resistant and recurrent tumors were associated with increased pro-inflammatory cytokines. Similarly, high metanephrine levels correlated with higher pro-tumoral cytokines. Epinephrine stimulation decreased CD8 + T-cell function in ascites of HGSOC patients. These data suggest a role for stress in immunosuppression and may impact efficacy of therapies that aim to restore T-cell function. 